\title{
Knowledge and beliefs towards universal safety precautions during the coronavirus disease (COVID-19) pandemic among the Indian public: a web-based cross-sectional survey
}

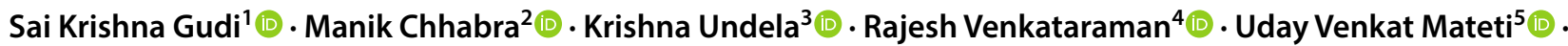 \\ Komal Krishna Tiwari ${ }^{6}$ (1) Sanath Nyamagoud ${ }^{7}$ (i)
}

Published online: 4 July 2020

(c) Springer Nature Switzerland AG 2020

\begin{abstract}
Background The novel coronavirus disease 2019 (COVID-19) is considered the most serious global health threat in recent times. As there is a current lack of approved treatments and vaccines, universal safety precautions (USPs) must be taken to deal with this emergency.

Objective The aim of this study was to assess the knowledge and beliefs of the Indian public with regard to USPs during the COVID-19 pandemic.

Methods A cross-sectional, web-based survey was conducted during March 2020. A 20-item self-administered questionnaire was developed, validated and distributed using Google Forms through social media networks. Binary logistic regression analysis was used to identify the factors influencing knowledge regarding COVID-19 USPs.

Results Of the 1117 individuals who participated in the survey, the mean age was $28.8 \pm 10.9$ years, $32.9 \%$ had a postgraduate education, $45 \%$ had a professional job, and $40 \%$ belonged to the upper-middle economic class. Overall, the mean correct response scores were $63 \%$ for USP knowledge and $83 \%$ for USP beliefs. All the sociodemographic variables were significantly $(p<0.001)$ associated with the USP knowledge levels. Importantly, students were less likely to have a lower level of USP knowledge compared with the other occupations (odds ratio $0.35,95 \%$ CI $0.23-0.53 ; p<0.001$ ).

Conclusion Although the knowledge and beliefs of the Indian public towards USPs are encouraging, there is a need for long-term educational interventions as the dynamics and severity of COVID-19 rapidly change. These findings could guide public health authorities to make and implement precautionary measures to combat this pandemic.
\end{abstract}

\section{Introduction}

The novel coronavirus 2019 (SARS-CoV-2) has caused the worldwide pandemic of coronavirus disease 2019 (COVID19). COVID-19 was initially identified as a cluster of pneumonia cases during late December 2019 in China [1] and rapidly spread worldwide. The definite modes of SARSCoV-2 transmission are not yet completely known; however, health officials suggest that it could primarily spread through droplets when an infected person coughs or sneezes,

Electronic supplementary material The online version of this article (https://doi.org/10.1007/s40267-020-00752-8) contains supplementary material, which is available to authorized users.

Sai Krishna Gudi

sknanu06@gmail.com; gudisk@myumanitoba.ca

Extended author information available on the last page of the article and by direct contact with infected individuals [2]. Unfortunately, no drugs or vaccines have been officially approved for the treatment of COVID-19, although some drugs, such as hydroxychloroquine and remdesivir, are under clinical investigation [3]. Therefore, acquiring and being adherent to the universal safety precautions (USPs) is the only method to control the spread of COVID-19. Implementing the following non-pharmacological USPs during the pandemic would relieve overloaded healthcare systems and the concerns of the public.

Maintaining personal hygiene is an essential practice to protect against any type of respiratory illness, including COVID-19. Hand washing and social/physical distancing are effective measures to prevent transmission between individuals $[4,5]$. Other major mitigating measures include isolation and quarantine, particularly of individuals with symptoms or confirmed COVID-19 cases. If the above-mentioned measures are insufficient in reducing the wide spread of the 
infection, community containment would be implemented where an entire community or neighbourhood is restricted in order to reduce personal interactions, except for inevitable situations. The use of personal protective equipment (PPE), such as face masks, gloves and hand sanitizers, is another precautionary control measure, although less effective than other control measures [6].

The course of COVID-19 in India initially seemed under control, with relatively lower rates of infection despite the large population; however, the number of COVID-19 cases and fatalities is increasing rapidly. Considering the dynamics of SARS-CoV-2, especially its higher rates of transmission, the COVID-19 outbreak in India could continue to worsen. Out of concern in this regard, this study was conducted to examine the knowledge and beliefs regarding COVID-19 USPs and mitigating strategies related to COVID-19 among the public of India.

\section{Methods}

\section{Study design}

A cross-sectional, web-based survey was conducted during March 2020 with the intention of obtaining responses regarding USPs and mitigating strategies towards COVID19 among the Indian public with access to the internet and/ or social media networks.

\section{Development of the questionnaire}

A 20-item semi-structured self-administered questionnaire was developed in the English language using fact sheets, course materials, information leaflets and booklets developed by the Health Protection Surveillance Centre (HPSC) [7], Public Health Agency (HSC) [8], National Health Service (NHS) [9], and the World Health Organization (WHO) [10] in relation to COVID-19 prevention. The study questionnaire included six demographic-related questions, followed by two domains, i.e. knowledge and beliefs, each with 10 questions. For the knowledge-related questions, all questions were multiple choice with three to four options, while for the belief-related questions, there were three multiple choice options (agree, disagree and do not know). The questionnaire mainly focused on aspects such as personal hygiene, proper handwashing, use of face masks, and social distancing techniques such as quarantine and isolation (Electronic Supplementary Material).

\section{Validity and reliability of the questionnaire}

The initial questionnaire was validated by subject experts (faculty members and researchers) using face and content validation methods. The questionnaire was checked for clarity, appropriateness, wordiness, and use of jargon and double-ended questions. The revised questionnaire was then assessed for reliability using split-half (internal reliability) and test-retest (external reliability) methods in a pilot study with 30 random respondents. The survey questions were split into two equal halves using the odd-even method and were distributed among the pilot study participants, where the responses between the two sets of questions (i.e. odd and even sets) were correlated. A reliability correlation coefficient of $\geq 0.7$ between the two sets of questions was considered to indicate reliability. The questionnaire had reliability correlation coefficients of 0.74 (split-half) and 0.72 (test-retest), and was revised based on the comments/feedback of participants in the pilot study.

\section{Sample size}

The study sample size was estimated using the Raosoft sample size calculator [11]. A minimum of 385 participants were required at a margin of error of 5\%, a $95 \%$ confidence interval (CI), and a population size of 1 billion at a $50 \%$ response distribution.

\section{Distribution of the questionnaire}

The final revised questionnaire was designed using Google forms [12] and was posted, advertised and distributed by the study investigators using their private accounts on social media platforms such as Facebook, WhatsApp, Telegram and LinkedIn. The public were requested to take part in the survey by completing the questionnaire without any time restrictions. Multiple responses or submissions were controlled using the 'Limit to one response' feature of Google forms.

\section{Ethical considerations}

The purpose of the survey was explained to potential participants, who were requested to provide consent of voluntary willingness prior to their participation. All procedures performed in this study involving human participants were in adherence to the ethics of the 1964 Helsinki declaration and its later amendments or comparable ethical standards. This study was conducted and reported according to the Checklist for Reporting Results of Internet E-Surveys (CHERRIES) guidelines [13].

\section{Statistical analysis}

Data were recorded in Microsoft Excel spreadsheets (Microsoft Corporation, Redmond, WA, USA) and cross-checked for accuracy. Statistical analysis was performed using IBM 
SPSS software, version 24 (IBM Corporation, Armonk, NY, USA) [14]. Descriptive statistics on sample characteristics were computed, including means with standard deviation, and frequency distributions. For assessing factors influencing USP knowledge, participants were categorized into low $(0-4)$ and high (5-10) knowledge score groups based on the cumulative scores. The factors influencing knowledge were identified using binary logistic regression analysis and represented as odds ratios with $95 \%$ CIs. $P$-values $<0.05$ were considered to be statistically significant.

\section{Results}

A total of 1117 participants completed the survey questionnaire. The mean age of study participants was $28.8 \pm 10.9$ years, with $>50 \%$ belonging to the $<25$ years age category. Participants were equally distributed by sex, and $>90 \%$ had either doctoral $(28.4 \%)$, postgraduate $(32.9 \%)$ or undergraduate $(30.1 \%)$ degrees as their educational status. Most participants were professional job holders ( $45 \%$ ) or students $(32 \%)$, and $\approx 40 \%$ belonged to the uppermiddle economic class $(26,355-52,733$ rupees/month) [see Table 1 for other sociodemographic characteristics of the study population].

\section{Knowledge regarding universal safety precautions (USPs)}

Although most of the study participants $(69.7 \%)$ provided the correct response with regard to the ideal length of time to wash hands in preventing the infection, almost half of the participants (46\%) were not aware of the ideal strength of the alcohol that a hand sanitizer should contain to be used during outbreaks, while more than two-thirds (70\%) did not know the ideal social-distancing distance. Around threequarters (74\%) and two-fifths (41\%) of participants were not aware of the quarantine and isolation procedures that are usually followed during outbreaks; however, most participants knew the correct answers regarding the self-isolation time period (84\%), type of face mask to be used (77\%), and social distancing procedures (94\%). The overall mean knowledge domain score was $63.0 \%$ (95\% CI 60.0-65.8), with a range of $25.6-93.6 \%$. Other knowledge domain scores are provided in Table 2.

\section{Beliefs regarding USPs}

Almost all study participants (94\%) believed that maintaining good personal hygiene, washing hands frequently using soap, avoiding handshaking behaviour, and avoiding placing fingers in the eyes, nose, and mouth would prevent the spread of the infection. Likewise, almost all (>96\%)
Table 1 Sociodemographic characteristics of 1117 study participants (questions $2-6$ on the questionnaire)

\begin{tabular}{|lc|}
\hline Variable & $\begin{array}{l}\text { No. of } \\
\text { participants } \\
(\%)\end{array}$ \\
\hline Age, years & \\
\hline$<25$ & $666(59.6)$ \\
\hline $25-45$ & $284(25.4)$ \\
\hline$>45$ & $167(15.0)$ \\
\hline Sex & \\
\hline Male & $568(50.9)$ \\
\hline Female & $549(49.1)$ \\
\hline Education level & \\
\hline Doctoral degree & $317(28.4)$ \\
\hline Postgraduate & $367(32.9)$ \\
\hline Undergraduate & $336(30.1)$ \\
\hline Post-secondary/diploma & $38(3.4)$ \\
\hline Secondary education (grades 9-12) & $48(4.3)$ \\
\hline High school (grades 5-8) & $11(1.0)$ \\
\hline Occupation status & \\
\hline Professional & $503(45.0)$ \\
\hline Skilled worker & $124(11.1)$ \\
\hline Unskilled worker & $46(4.1)$ \\
\hline Unemployed & $13(1.21)$ \\
\hline Retired employee & $53(471)$ \\
\hline Homemaker & $21(1.9)$ \\
\hline Student & $357(32.0)$ \\
\hline Economical class (rupees/month) & $272(24.4)$ \\
\hline Upper ( 52,734$)$ & $445(39.8)$ \\
\hline Upper-middle (26,355-52,733) & $45(4.0)$ \\
\hline Lower-middle (19,759-26354) & \\
\hline Upper-lower (13,161-19,758) & \\
\hline Lower ( $\leq 13,160)$ & \\
\hline (26.4)
\end{tabular}

believed that adhering to social distancing measures and staying at home would be good practices in combating the spread. However, three-quarters of participants believed that wearing a face mask is considered appropriate and protective even in the absence of symptoms. The overall mean 'beliefs' domain score was 83.0\% (95\% CI 80.7-85.1), with a range of $25.3-96.4 \%$. Other domain data regarding beliefs are provided in Table 3.

\section{Factors influencing the knowledge of study participants}

Based on the survey responses, participants had considerably true beliefs, as almost all questions had more than a $90 \%$ correct response rate. Therefore, we decided to only assess the factors influencing knowledge as there was no 
Table 2 Knowledge of universal safety precautions in 1117 study participants

Question (questions 7-16 on the questionnaire)

Correct answer No. of responses (\%)

Correct Incorrect

7. What would be the ideal length of time to wash hands in preventing the spread of COVID-19?

$20 \mathrm{~s}$

779 (69.7) $338(30.3)$

8. What is the ideal strength of alcohol that a hand sanitizer should contain to be used during outbreaks?

9. What would be the ideal distance to be maintained in preventing the spread of COVID-19?

10. During outbreaks, 'quarantine' is a procedure usually followed by?

11. During outbreaks, 'isolation' is a procedure usually followed by?

12. Health status of all the close contacts should be monitored for how many days from the last exposure (i.e. self-isolation period)?

13. Which type of face mask is considered as ideally protective during outbreaks?

14. Social distancing means staying away from the crowd and maintaining minimum distance from True people around, with the intention of minimizing the transmission of an outbreak?

15. The term 'close contacts' are those who had provided care for infected persons? True

16. The term 'transient contacts' are those who had interacted with the infected persons for a short True period of time?
$60 \%$

$603(54.0) \quad 514(46.0)$

$2 \mathrm{~m} / 6 \mathrm{ft}$

$338(30.3) \quad 779(69.7)$

At-risk people

$286(25.6) \quad 831(74.4)$

Infected people

$654(58.5) \quad 463(41.5)$

14 days

$940(84.2) \quad 177(15.8)$

$1045(93.6) \quad 72(6.4)$

$705(63.1) \quad 412(36.9)$

$821(73.5) \quad 296(26.5)$
N-95 face mask $857(76.7) \quad 260(23.3)$

All questions were multiple choice with three (true, false and not sure) or four possible options

Table 3 Beliefs regarding universal safety precautions in 1117 study participants

\begin{tabular}{|c|c|c|c|}
\hline \multirow[t]{2}{*}{ Question (questions $17-26$ on the questionnaire) } & \multirow[t]{2}{*}{ Correct answer } & \multicolumn{2}{|c|}{ No. of responses $(\%)$} \\
\hline & & Correct & Incorrect \\
\hline $\begin{array}{l}\text { 17. Maintaining good personal hygiene and being socially responsible would prevent the spread of } \\
\text { COVID-19? }\end{array}$ & Agree & 1045 (93.6) & $72(6.4)$ \\
\hline 18. Washing hands frequently using soap or sanitizer would prevent the spread of COVID-19? & Agree & 1045 (93.6) & $72(6.4)$ \\
\hline 19. Avoiding handshaking behaviour would prevent the spread of COVID-19? & Agree & 1047 (93.7) & $70(6.3)$ \\
\hline 20. Avoiding placing fingers into eyes, nose and mouth would prevent the spread of COVID-19? & Agree & $1050(94.0)$ & $67(6.0)$ \\
\hline $\begin{array}{l}\text { 21. Coughing and sneezing into the elbow or within the clothing is a good practice in preventing the } \\
\text { spread of COVID-19? }\end{array}$ & Agree & $961(86.0)$ & $156(14.0)$ \\
\hline $\begin{array}{l}\text { 22. Limiting eating and sharing food with colleagues and friends would prevent the spread of } \\
\text { COVID-19? }\end{array}$ & Agree & $745(66.7)$ & $372(33.3)$ \\
\hline $\begin{array}{l}\text { 23. Following social distancing measures and avoiding crowded places would limit the spread of } \\
\text { COVID-19? }\end{array}$ & Agree & $1076(96.3)$ & $41(3.7)$ \\
\hline $\begin{array}{l}\text { 24. Wearing a face mask is considered appropriate and protective, although not having any symp- } \\
\text { toms? }\end{array}$ & Disagree & $283(25.3)$ & $834(74.7)$ \\
\hline $\begin{array}{l}\text { 25. Proper use of a face mask should include covering nose, mouth and chin with the coloured side } \\
\text { facing outside? }\end{array}$ & Agree & $978(87.6)$ & $139(12.4)$ \\
\hline 26. Staying at home would play a significant role in preventing the spread of COVID-19? & Agree & 1077 (96.4) & $40(3.6)$ \\
\hline
\end{tabular}

All questions were multiple choice questions with three possible options (agree, disagree and do not know)

need to identify the predictors for beliefs, based on our preliminary analysis. Binary logistic regression analyses indicated that increasing age, female sex, low level of education, non-professional occupation and lower economic class were the factors responsible for the low level of USP knowledge. Of note, participants with an occupational status of 'student' were less likely to have lower scores [odds ratio (OR) $0.35,95 \%$ CI $0.23-0.53 ; p<0.001]$ than professionals (Table 4).

\section{Discussion}

This study appears to be the first to assess the knowledge and beliefs towards USPs in combating the spread of COVID-19 in the Indian public. Our results noted that participant knowledge regarding the self-isolation time periods and the social distancing measures were relatively high, whereas other knowledge (ideal length of time to wash hands, the strength of alcohol that a hand sanitizer 
Table 4 Factors influencing knowledge of universal safety precautions in 1117 study participants

\begin{tabular}{|c|c|c|c|c|}
\hline \multirow[t]{2}{*}{ Sociodemographic factor } & \multirow[t]{2}{*}{ No. of participants } & \multicolumn{2}{|c|}{ No. of participants (\%) } & \multirow[t]{2}{*}{ OR $(95 \% \mathrm{CI})$} \\
\hline & & Low score $(0-4)$ & High score $(5-10)$ & \\
\hline \multicolumn{5}{|l|}{ Age, years } \\
\hline$<25$ & 666 & $149(22.4)$ & $517(77.6)$ & Reference \\
\hline $25-45$ & 284 & $130(45.8)$ & $154(54.2)$ & $2.93(2.18-3.94)^{* *}$ \\
\hline$>45$ & 167 & $69(41.3)$ & $98(58.7)$ & $2.44(1.71-3.49)^{* *}$ \\
\hline \multicolumn{5}{|l|}{ Sex } \\
\hline Male & 568 & $109(19.2)$ & $459(80.8)$ & Reference \\
\hline Female & 549 & $238(43.3)$ & $311(56.7)$ & $3.22(2.46-4.22)^{* *}$ \\
\hline \multicolumn{5}{|l|}{ Education level } \\
\hline Doctoral degree & 317 & $18(5.7)$ & $299(94.3)$ & Reference \\
\hline Postgraduate & 367 & $41(11.2)$ & $326(88.8)$ & $2.09(1.17-3.72)^{*}$ \\
\hline Undergraduate & 336 & $38(11.3)$ & $298(88.7)$ & $2.12(1.18-3.80)$ \\
\hline Post-secondary/diploma & 38 & $20(52.6)$ & $18(47.4)$ & $18.46(8.34-40.86)^{* *}$ \\
\hline Secondary education (grades 9-12) & 48 & $27(56.2)$ & $21(43.8)$ & $21.34(10.16-44.88)^{* *}$ \\
\hline High school (grades 5-8) & 11 & $5(45.4)$ & $6(54.6)$ & $13.84(3.85-49.72)^{* *}$ \\
\hline \multicolumn{5}{|l|}{ Occupational status } \\
\hline Professional & 503 & $114(22.7)$ & $389(77.3)$ & Reference \\
\hline Skilled worker & 124 & $46(37.1)$ & $78(62.9)$ & $2.01(1.32-3.06)^{* *}$ \\
\hline Unskilled worker & 46 & $31(67.4)$ & $15(32.6)$ & $7.05(3.68-13.52)^{* *}$ \\
\hline Unemployed & 13 & $9(69.2)$ & $4(30.8)$ & $7.68(2.32-25.39) * *$ \\
\hline Retired employee & 53 & $31(58.5)$ & $22(41.5)$ & $4.81(2.68-8.63)^{* *}$ \\
\hline Homemaker & 21 & $14(66.7)$ & $7(33.3)$ & $6.83(2.69-17.31)^{* *}$ \\
\hline Student & 357 & $33(9.2)$ & $324(90.8)$ & $0.35(0.23-0.53)^{* *}$ \\
\hline \multicolumn{5}{|l|}{ Economic class (rupees/month) } \\
\hline Upper $(\geq 52,734)$ & 272 & $83(30.5)$ & $189(69.5)$ & Reference \\
\hline Upper-middle $(26,355-52,733)$ & 44 & $117(26.3)$ & $328(73.7)$ & $0.81(0.58-1.13)$ \\
\hline Lower-middle $(19,759-26,354)$ & 45 & $32(71.1)$ & 13 (28.9) & $5.61(2.80-11.22)^{* *}$ \\
\hline Upper-lower $(13,161-19,758)$ & 295 & $192(65.1)$ & $103(34.9)$ & $4.25(2.99-6.04)^{* *}$ \\
\hline Lower $(\leq 13,160)$ & 60 & $39(65.0)$ & $21(35.0)$ & $4.23(2.34-7.63)^{* *}$ \\
\hline
\end{tabular}

$O R$ odds ratio, $C I$ confidence interval

${ }^{*} p \leq 0.011, * * p \leq 0.001$

should contain to be used during outbreaks, ideal distance to be maintained in preventing the spread of COVID-19, quarantine and isolation procedures, type of face mask to be used, and the difference between close and transient contacts) was insufficient. Overall, correct responses for USP beliefs were relatively high, except the responses for questions regarding eating and sharing food with colleagues and friends during the COVID-19 pandemic and wearing a face mask without having any symptoms. Overall, this indicates that educational interventions regarding USP for COVID-19 are needed for the general public in India.

USPs and community mitigation strategies, such as maintaining personal hygiene, handwashing behaviour, social distancing, isolation and quarantine methods, and rational use of face masks, could play a major role in diminishing
SARS-CoV-2 transmission. Generally, educating the public, with an emphasis on personal hygiene such as washing hands, would take a lot of time and effort, especially in developing countries such as India. A large cross-sectional comparative study conducted in Bangladesh [15] found a significant gap between perceptions and the practice of proper handwashing behaviours. It found that handwashing behaviour with soap before eating food was lower at baseline than at the end of follow-up ( $8 \%$ vs $22 \%$ ). Furthermore, socioeconomic status, including education, has shown a positive association with handwashing, which is similar to our study findings [15].

In contrast with our results, a study conducted during an outbreak of the H1N1 influenza-A pandemic in 2009 at a large public university in the USA found poor compliance with the mitigation strategies, such as staying at home while 
ill, in avoiding the spread of the virus. In addition, around half of the study participants, including students, staff and faculty members, had attended social gatherings despite acute respiratory infection and feeling ill [16]. Among various infection control strategies, the use of PPE is essential during outbreaks such as COVID-19, especially among healthcare workers and infected people. Although PPE is not recommended as a frontline defence measure, it should be included with other administrative and environmental control measures.

Unfortunately, a recent systematic review regarding the use of PPE to protect against respiratory infections in Pakistan found that PPE was not available at many facilities, and its use was limited during high-risk situations [17]. In addition, the systematic review observed low compliance towards PPE among healthcare workers in relation to the reuse behaviour of PPE. In another cross-sectional survey that evaluated the knowledge, use and barriers towards the use of PPE for airway management among emergency medical technicians during the SARS outbreak in Canada, most study participants (91.5\%) thought $\mathrm{N}-95$ respirator masks were the safest and should be used during outbreaks [6], which is in keeping our study. However, the appropriate selection and efficacy of the respiratory protective apparatus has always been controversial [18]. The current study findings related to the proper use of a face mask (question 25) are in accordance with a cross-sectional study that assessed the use of face masks in a primary care outpatient setting in Hong Kong, where more than half of the participants (52\%) demonstrated the correct steps in wearing a face mask [19].

Implementing mitigation strategies such as isolation, quarantine and community containment during outbreaks would be associated with major challenges, such as early case detection in order to self-isolate, the need for psychological support, availability of basic needs while being separated from the public, and, most importantly, dealing and managing with the ethical codes, principles, self-determination, individual conflicts and rights of liberty of the public. Such challenges should be considered when implementing strategies during pandemics such as COVID-19. In a recent epidemic network model by Leung et al., it was inferred that social distancing during an epidemic might have potential negative effects, such as losing social contacts, at the population level [20]. The authors also stated that rational preventive measures during epidemics could have a countereffect on the population in the long run, as such measures might result in behavioural changes among the public, which could further worsen epidemic outcomes. However, policies and decision making regarding mitigation strategies should not be generalized considering the dynamics of other pandemics such as influenza, Middle East respiratory syndrome (MERS) and severe acute respiratory syndrome (SARS).
Looking at the current role of USPs and social distancing measures in combating the spread of COVID-19, it is worthwhile and wise to implement and adhere to such strategies in a timely manner. In this regard, the UK government has changed its action plan in dealing with COVID-19 following a modelling study that estimated 260,000 potential deaths [21]. As a result, a combination of social distancing of the entire population, home isolation of infected cases, quarantining family members of infected individuals, and possible school and university closures were implemented to try to reduce the number of potential deaths [22]. Lastly, the COVID-19 pandemic has taught government bodies and healthcare sectors how to deal with this outbreak and how to be prepared to face future outbreaks of infectious diseases [23]. Research focusing on the transmission dynamics of SARS-CoV-2 is warranted to improve prevention strategies.

\section{Study limitations}

Certain limitations should be considered when interpreting the findings of this study. First, as this was a cross-sectional survey, causal inferences cannot be made, and chances for recall and information bias may exist. Second, as the questionnaire was self-administered, and was thus dependent on the self-reported data, it is difficult to predict and understand whether the respondents completed the survey honestly (i.e. social desirability bias and the responses provided by participants may not reflect reality). Lastly, as this was an internet-based online survey, it might not capture the responses from those regions with restricted access to social media. This may introduce demographic, sampling and coverage selection bias, especially as responses were mostly from a relatively young, educated, professional and internet-active population. Therefore, the study findings could not be generalized to the country's entire population.

\section{Take home messages}

- Studies on knowledge, attitudes, perceptions and beliefs towards preventive and mitigating strategies during outbreaks and pandemics, especially in countries with large populations, are needed.

- Focusing on USPs, such as maintaining personal hygiene, washing hands, adhering to physical distancing techniques, self-isolation practices, and use of face masks and hand sanitizers, among the general public would significantly contribute to reducing the transmission of COVID-19.

- Although the overall level of USP knowledge and beliefs among the Indian public during the COVID-19 pandemic was encouraging, there is a need for long-term educa- 
tional interventions, as the current scenario is rapidly changing.

- Based on the findings of this study, educational interventions should mainly target populations who are aged or female, have low levels of education or socioeconomic status, or hold a non-professional occupation.

- The findings of this study could help guide public health authorities in making and implementing strategies to combat COVID-19; however, the assessment of factors influencing compliance with USP measures is warranted.

Acknowledgements The authors would like to extend their gratitude towards all study participants for investing their time, interest and voluntary participation in providing the essential information.

Author contributions SKG conceived the idea, designed the study and drafted the manuscript; SKG, MC and KU developed the survey instrument, and collected and analyzed the data; and RV, UVM, SN and KKT contributed towards the reliability and validity of the questionnaire, pilot testing, distribution of the survey questionnaire and proofreading the manuscript. All authors approved the version submitted for publication.

\section{Compliance with ethical standards}

Funding No funding was received.

Conflict of interest Sai Krishna Gudi, Manik Chhabra, Krishna Undela, Rajesh Venkataraman, Uday Venkat Mateti, Komal Krishna Tiwari and Sanath Nyamagoud declare no conflicts of interest.

\section{References}

1. Zhu N, Zhang D, Wang W, et al. A novel coronavirus from patients with pneumonia in China, 2019. N Engl J Med. 2020;382(8):727-33.

2. Li Q, Guan X, Wu P, et al. Early transmission dynamics in Wuhan, China, of novel coronavirus-infected pneumonia. N Engl J Med. 2020;382(13):1199-207.

3. Liu J, Cao R, Xu M, et al. Hydroxychloroquine, a less toxic derivative of chloroquine, is effective in inhibiting SARS-CoV-2 infection in vitro. Cell Discov. 2020;6:16

4. Aiello AE, Coulborn RM, Perez V, et al. Effect of hand hygiene on infectious disease risk in the community setting: a meta-analysis. Am J Public Health. 2008;98(8):1372-81.

5. Qualls N, Levitt A, Kanade N, et al. Community mitigation guidelines to prevent pandemic influenza: United States, 2017. MMWR Recomm Rep. 2017;66(1):1-34.
6. Visentin LM, Bondy SJ, Schwartz B, et al. Use of personal protective equipment during infectious disease outbreak and nonoutbreak conditions: a survey of emergency medical technicians. CJEM. 2009;11(1):44-56.

7. Health Protection Surveillance Centre. COVID-19 posters. https:// www.hpsc.ie/a-z/respiratory/coronavirus/novelcoronavirus/poste rs/. Accessed 10 May 2020.

8. Public Health Agency. Public information posters and leaflets for download. https://www.publichealth.hscni.net/news/covid-19coronavirus\#public-information-posters-and-leaflets-for-downl oad. Accessed 10 May 2020.

9. National Health Service. Coronavirus (COVID-19). https://www. nhs.uk/conditions/coronavirus-covid-19/. Accessed 10 May 2020.

10. World Health Organization. Coronavirus disease (COVID-19) training: online training. https://www.who.int/emergencies/disea ses/novel-coronavirus-2019/training/online-training. Accessed 10 May 2020.

11. Raosoft. Sample size calculator. http://www.raosoft.com/sampl esize.html. Accessed 28 Feb 2020.

12. Google Forms. Survey questionnaire. https://forms.gle/a6nHm QjdWzbXVLa56. Accessed 10 May 2020.

13. Eysenbach G. Improving the quality of web surveys: the checklist for reporting results of internet e-surveys (CHERRIES). J Med Internet Res. 2004;6(3):e34.

14. IBM Corporation. IBM SPSS statistics for windows, 2016. Version 24.0. Armonk: IBM Corporation; 2016.

15. Rabbi SE, Dey NC. Exploring the gap between hand washing knowledge and practices in Bangladesh: a cross-sectional comparative study. BMC Public Health. 2013;13:89.

16. Mitchell T, Dee DL, Phares CR, et al. Non-pharmaceutical interventions during an outbreak of 2009 pandemic influenza A (H1N1) virus infection at a large public university, April-May 2009. Clin Infect Dis. 2011;52(Suppl 1):S138-45.

17. Chughtai AA, Khan W. Use of personal protective equipment to protect against respiratory infections in Pakistan: a systematic review. J Infect Public Health. 2019;12(4):522-7.

18. Chung SJ, Ling ML, Seto WH, et al. Debate on MERS-CoV respiratory precautions: surgical mask or N95 respirators? Singap Med J. 2014;55(6):294-7.

19. Ho HS. Use of face masks in a primary care outpatient setting in Hong Kong: knowledge, attitudes and practices. Public Health. 2012;126(12):1001-6.

20. Leung KY, Ball F, Sirl D, et al. Individual preventive social distancing during an epidemic may have negative population-level outcomes. J R Soc Interface. 2018;15(145):20180296.

21. Mahase E. Covid-19: UK starts social distancing after new model points to 260000 potential deaths. BMJ. 2020;368:m1089.

22. Undela K, Gudi SK. Assumptions for disparities in case-fatality rates of coronavirus disease (COVID-19) across the globe. Eur Rev Med Pharmacol Sci. 2020;24(9):5180-2.

23. Gudi SK, Tiwari KK. Preparedness and lessons learned from the novel coronavirus disease. Int J Occup Environ Med. 2020;11(2):108-12. 


\section{Affiliations}

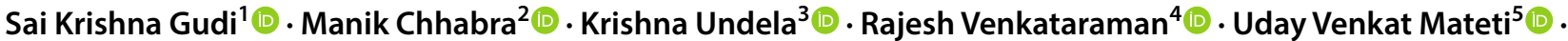 Komal Krishna Tiwari ${ }^{6}$ (D) Sanath Nyamagoud ${ }^{7}$ (i)}

Manik Chhabra

manikchhabra57@gmail.com

Krishna Undela

krishnaundela@gmail.com

Rajesh Venkataraman

rajeshvenky_research@hotmail.com

Uday Venkat Mateti

udayvenkatmateti@gmail.com

Komal Krishna Tiwari

komalkrishnatiwari@gmail.com

Sanath Nyamagoud

dr.sanathnyamagoud@gmail.com

1 College of Pharmacy, Rady Faculty of Health Sciences,

University of Manitoba, 228 Notre Dame Avenue, Winnipeg, MB R3B 1N7, Canada
2 Department of Pharmacy Practice, Indo-Soviet Friendship College of Pharmacy, Moga, Punjab, India

3 Department of Pharmacy Practice, JSS College of Pharmacy, JSS Academy of Higher Education AND Research, Mysore, Karnataka, India

4 Department of Pharmacy Practice, Sri Adichunchanagiri College of Pharmacy, Adichunchanagiri University, B.G. Nagara, Karnataka, India

5 Department of Pharmacy Practice, NGSM Institute of Pharmaceutical Sciences, NITTE (Deemed to be University), Mangalore, Karnataka, India

6 JSS College of Physiotherapy, Rajiv Gandhi University of Health Sciences, Mysore, Karnataka, India

7 Department of Pharmacy Practice, KLE University, Hubli, Karnataka, India 